\title{
Computation of the spectrum of spatial Lyapunov exponents for the spatially extended beam-plasma systems and electron-wave devices
}

\author{
Alexander E. Hramov, Alexey A. Koronovskii, Vladimir A. Maximenko, and Olga I. Moskalenko \\ Faculty of Nonlinear Processes, Saratov State University, \\ Astrakhanskaya str., 83, Saratov, 410012, Russia
}

(Dated: November 6, 2018)

\begin{abstract}
The spectrum of Lyapunov exponents is powerful tool for the analysis of the complex system dynamics. In the general framework of nonlinear dynamical systems a number of the numerical technics have been developed to obtain the spectrum of Lyapunov exponents for the complex temporal behavior of the systems with a few degree of freedom. Unfortunately, these methods can not apply directly to analysis of complex spatio-temporal dynamics in plasma devices which are characterized by the infinite phase space, since they are the spatially extended active media. In the present paper we propose the method for the calculation of the spectrum of the spatial Lyapunov exponents (SLEs) for the spatially extended beam-plasma systems. The calculation technique is applied to the analysis of chaotic spatio-temporal oscillations in three different beam-plasma model: (1) simple plasma Pierce diode, (2) coupled Pierce diodes, and (3) electron-wave system with backward electromagnetic wave. We find an excellent agreement between the system dynamics and the behavior of the spectrum of the spatial Lyapunov exponents. Along with the proposed method, the possible problems of SLEs calculation are also discussed. It is shown that for the wide class of the spatially extended systems the set of quantities included in the system state for SLEs calculation can be reduced using the appropriate feature of the plasma systems.
\end{abstract}

PACS numbers: 05.45.-a, 05.10.-a

Keywords: spectrum of Lyapunov exponents, spatially extended systems, beam plasma systems, electron wave media, Pierce diode, transverse field backward wave oscillator, reference system state.

\section{Introduction}

Nonlinear dynamics and chaos in plasma devices and microwave systems with interacted electron and electromagnetic waves is often observed in bounded plasmas and electron and ions streams, where the nonlinearity and plasma instabilities are combined with dissipation caused by current flow to the boundary electrodes. Therefore, the development of the methods of analysis of spatially extended beam-plasma systems is a very important problem due to such applications of chaos theory in plasma physics as the identification of chaoti spatial-temporal oscillations in microwave and plasma devices (e.g. free electron lasers, pasotrons, vircators, etc) 1 [3], chaos control and suppression of turbulence [4 8], and chaotic synchronization in the plasma and microwave electronic systems [9 12 .

The Lyapunov exponents are powerful and useful tool for the complex system dynamics to be analyzed. They are used widely to characterize the systems with small number of degrees of freedom in the different fields of science 13 16, such as physics, physiology and medicine, molecular dynamics, astronomy, etc.

At the same, the majority of the plasma systems are characterized by the infinite phase space, since they are the spatially extended active media. Unfortunately, the direct application of the Lyapunov exponent calculation technique developed for the system with the small number of degrees of freedom to the objects of the beamplasma and electron-wave systems being the spatially extended systems is problematic. The main causes of the this problem are the following: (i) the phase space of the spatially distributed system is infinite; (ii) the number of the Lyapunov exponents is also infinite; (iii) the system state (defined for each type of the system under study in its own way) must be used instead of the finite-dimensional vector; (iv) the orthogonalizaiton and normalization procedures based on Gram-Schmidt orthonormalization must be modified to be applicable for the states.

Nevertheless, the scientists try to use the Lyapunov exponents for the spatially extended systems due to the great efficiency of this tool. Mainly, all attempts are reduced to the use of the modifications of the standard methods developed for the finite dimensional systems (e.g., the estimation of the highest Lyapunov exponent from time series or the representation of the spatially extended system as the finite dimensional one with the high dimension by means of the discretization). Unfortunately, these approaches do not take into account the distinguishing characteristics of the spatially extended systems and have serious limitations connected with the high dimension of the phase space of the discretized system

Recently, the successful attempt to compute the highest Lyapunov exponent taking into account the core features of the spatially extended systems has been made for plasma system models [5, 12]. In the present work we develop the technique of the spectrum of the spatial Lyapunov exponents (SLEs) calculation for the distributed beam-plasma systems and electron-wave devices. The structure of the article is the following. In Sec. 1 the general idea of the proposed approach is given. The next Sec. II contains the results concerning the computation 
of the spectrum of spatial Lyapunov exponents for the spatially extended beam plasma system. As the model system under study the Pierce diode has been selected, with the spectrum of Lyapunov exponents being calculated both for autonomous oscillator and for two coupled plasma diodes. To illustrate the generality and efficiency of the proposed method, in Sec. III the SLEs have been also calculated for the transverse field backward wave oscillator being the classical models of the electrons-wave systems. The problem concerning the selection of the system state for the SLEs computation is discussed in Sec. IV] The final remarks are given in Conclusion.

\section{THEORY OF THE SPATIAL LYAPUNOV EXPONENTS}

The main idea of the computation of the spectrum of the spatial Lyapunov exponents is the following. Let

$$
\hat{L}(\mathbf{U}(x, t))=0
$$

be an operator determining the evolution of the spatially extended system under study, $\mathbf{U}(x, t)$ is the system state which (in the general case) depends on the spatial coordinate $x \in[0, L]$ and time $t$.

The state $\mathbf{U}(x, t)$ plays the role of the reference point to study the evolution of the small perturbations. To compute $N$ largest spatial Lyapunov exponents we consider the set of perturbations $\mathbf{V}_{i}(x, t), i=1, \ldots, N$ which are orthogonal

$$
\left(\mathbf{V}_{i}, \mathbf{V}_{j}\right)= \begin{cases}1, & i=j \\ 0, & i \neq j\end{cases}
$$

where $(\mathbf{U}, \mathbf{V})$ is the scalar product. If the system state is the $N_{d}$-dimensional vector $\mathbf{U}=\left(u_{1}, \ldots, u_{N_{d}}\right)^{T}$ or real function $\mathbf{U}=u(x, t)$ defined in $\mathbb{L}^{2}$ the definition of the scalar product is

$$
(\mathbf{U}, \mathbf{V})=\sum_{i=1}^{N_{d}} u_{i}(t) v_{i}(t)
$$

or

$$
(\mathbf{U}, \mathbf{V})=\int_{L} u(x, t) v(x, t) d x
$$

respectively. At the same time, the state of the spatially extended beam-plasma system should be usually defined in a more complicated way, and, as a consequence, the definition of the scalar product is also more complex. E.g., if the system state is the $N_{d}$-dimensional vectorfunction $\mathbf{U}(x, t)=\left(u_{1}(x, t), \ldots, u_{N_{d}}(x, t)\right)^{T}$ the scalar product should be defined as

$$
(\mathbf{U}, \mathbf{V})=\sum_{i=1}^{N_{d}} \int_{L} u_{i}(x, t) v_{i}(x, t) d x
$$

Additionally to the orthogonality requirement (2) all perturbations $\mathbf{V}_{i}(x, t)$ must also obey the normalization condition

$$
\left\|\mathbf{V}_{i}(x, 0)\right\|=1
$$

where $\|\mathbf{V}\|=\sqrt{(\mathbf{V}, \mathbf{V})}$.

The set of the perturbations $\mathbf{V}_{i}(x, t)$ fulfilling requirements (2) and (6) may be obtained with the help of the Gramm-Schmidt orthonormalization method

$$
\left\{\begin{array}{l}
\mathbf{V}_{i}=\frac{\tilde{\mathbf{V}}_{i}(x, 0)}{\left\|\tilde{\mathbf{V}}_{i}(x, 0)\right\|}=\frac{\tilde{\mathbf{V}}_{i}(x, 0)}{\sqrt{\left(\tilde{\mathbf{V}}_{i}(x, 0), \tilde{\mathbf{V}}_{i}(x, 0)\right)}} \\
\tilde{\mathbf{V}}_{1}(x, 0)=\varphi_{1}(x) \\
\tilde{\mathbf{V}}_{i+1}(x, 0)=\varphi_{i+1}(x)-\sum_{k=1}^{i}\left(\mathbf{V}_{k}, \varphi_{i+1}\right) \mathbf{V}_{k}(x, 0),
\end{array}\right.
$$

$i=1,2, \ldots, N-1$, where $\varphi_{1}(x), \varphi_{2}(x), \ldots, \varphi_{N}(x)$ is the set of the linearly independent arbitrary states defined for the system under study.

To compute the spectrum of the spatial Lyapunov exponents of the spatially extended system the behavior both of the state $\mathbf{U}(x, t)$ of the system under study and all perturbations $\mathbf{V}_{i}(x, t)(i=1,2, \ldots, N-1)$ has to be considered. The evolution of the system state $\mathbf{U}(x, t)$ is defined by Eq. (1), whereas the development of the perturbations $\mathbf{V}_{i}(x, t)$ of the reference state is described by the linearization

$$
\partial \hat{L}\left(\mathbf{U}(x, t), \mathbf{V}_{i}(x, t)\right)=0
$$

of the evolution operator (11) applied in the vicinity of the state $\mathbf{U}(x, t)$.

After the time interval with the length $T$ the obtained set of the perturbations $\mathbf{V}_{i}(x, T)$ must be orthogonalized and normalized with the help of the Gramm-Shmidt method again, with the set of the functions $\varphi_{i}(x)$ being the considered perturbations $\mathbf{V}_{i}(x, T)$. In other words,

$$
\varphi_{i}(x)=\mathbf{V}_{i}(x, T) \text {. }
$$

The described algorithm for spatially extended system must be repeated many times. After $M$ iterations the Lyapunov sums are calculated

$$
S_{i}=\sum_{j=1}^{M} \ln \left\|\tilde{\mathbf{V}}_{i}(x, j T)\right\|,
$$

where the perturbations before renormalization but after orthogonalizaiton are used. The spatial Lyapunov exponents are estimated as

$$
\Lambda_{i}=\frac{S_{i}}{M T}
$$

\section{SPECTRUM OF SPATIAL LYAPUNOV EXPONENTS FOR PIERCE DIODE}

To illustrate the efficiency of the proposed method for the computation of spatial Lyapunov exponents we consider the behavior of autonomous and coupled Pierce 


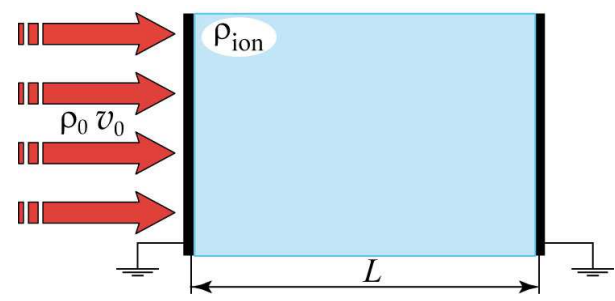

FIG. 1: (Color online) Schematic diagram of Pierce diode

diodes [17] being the classical models of beam-plasma systems, demonstrating the complex spatio-temporal oscillations including the chaotic ones [17 19$]$.

\section{A. Pierce diode}

A Pierce diode [17 20] is schematically illustrated in Fig. 1] and consists of two infinite parallel plains pierced by a mono-energetic electron beam. Grids are grounded, with the distance between them being $L$. The charge density $\rho_{0}$ and electron velocity $v_{0}$ are constant at the system input. The region between two plains is uniformly filled by neutralizing stationary ions, with the density $\left|\rho_{\text {ion }}\right|$ being equal to the non-perturbed electron beam density $\left|\rho_{0}\right|$.

The dimensionless Pierce parameter $\alpha=\omega_{p} L / v_{0}$ determines the dynamics of the system (here $\omega_{p}$ is the electron beam plasma frequency, $v_{0}$ is the non-perturbed electron velocity, $L$ is the distance between the diode plains). With $\alpha>\pi$ the so-called Pierce instability is developed in the system and a virtual cathode is formed in the electron beam [19]. A detailed analysis of the Pierce instability is provided in Ref. [21].

Here we focus our attention on a narrow range of Pierce parameter near $\alpha \sim 3 \pi$ where the increase of the instability is suppressed by the nonlinearity, and a reflectionless regime takes place in the electron beam [19, 20]. In this case the system behavior may be described by the fluid equations [18 20], that give rise to various types of beamplasma chaotic oscillations [5, 18, 19, 22].

The dynamics of the electron flow in the Pierce diode (in the fluid electronic approximation) is described by the self-congruent system of dimensionless Poisson, continuity and momentum equations

$$
\begin{gathered}
\frac{\partial^{2} \varphi}{\partial x^{2}}=-\alpha^{2}(\rho-1), \\
\frac{\partial \rho}{\partial t}+v \frac{\partial \rho}{\partial x}+\rho \frac{\partial v}{\partial x}=0, \\
\frac{\partial v}{\partial t}+v \frac{\partial v}{\partial x}=-\frac{\partial \varphi}{\partial x}
\end{gathered}
$$

with the boundary conditions

$$
v(0, t)=1, \quad \rho(0, t)=1, \quad \varphi(0, t)=\varphi(1, t)=0,
$$

where $\varphi(x, t)$ is the dimensionless potential of the electric field, $\rho(x, t)$ and $v(x, t)$ are the dimensionless density and velocity of the electron beam $(0 \leq x \leq 1)$, respectively. The dimensional variables $\left(\varphi^{\prime}, \rho^{\prime}, v^{\prime}, x^{\prime}, t^{\prime}\right)$ are connected with the dimensionless ones as $\varphi^{\prime}=\left(v_{0}^{2} / \eta\right) \varphi, \rho^{\prime}=\rho_{0} \rho$, $v^{\prime}=v_{0} v, x^{\prime}=L x, t^{\prime}=\left(L / v_{0}\right) t$, where $\eta$ is the specific electron charge, $v_{0}$ and $\rho_{0}$ are the non-perturbed velocity and density of the electron beam.

The system (12)-(14) with the boundary conditions (15) describes the dynamics of electron flow in the autonomous Pierce diode. It has been solved numerically using finite difference approximation. Numerical solutions for motion (14) and continuity (13) equations have been found by means of the explicit scheme with the differences against flow, and Poisson equation (12) has been integrated using the error vector propagation method 23]. The time and space integration steps have been selected as $\Delta x=0.005$ and $\Delta t=0.003$, respectively.

As a vector state of Pierce diode we have used the following one

$$
\mathbf{U}=(\rho(x, t), v(x, t))^{T} .
$$

In this case, according to (5), the scalar product is defined as

$$
\left(\mathbf{U}_{1}, \mathbf{U}_{\mathbf{2}}\right)=\int_{0}^{1}\left[\rho_{1}(x, t) \rho_{2}(x, t)+v_{1}(x, t) v_{2}(x, t)\right] d x,
$$

where the integration in (17) should be performed over a whole length of the considered system. Note, that the potential $\varphi(x, t)$ is not included in the vector state $\mathbf{U}$ since it could be explicitly defined through the density of the electron beam $\rho(x, t)$ by Poisson equation (12) and boundary conditions (15). In other words, to explicitly define the Pierce diode system state it is sufficient to know the distributions of the density $\rho(x, t)$ of the electron beam and velocity $v(x, t)$. It allows us, using the evolution operator $\hat{L}(\mathbf{U})$, i.e. the system (12)-(14) with boundary conditions (15), to calculate the analogues states in the other moments of time.

As the perturbations $\mathbf{V}_{i}$ we have used the vectors

$$
\mathbf{V}_{i}=\left(\xi_{i}^{\rho}(x, t), \xi_{i}^{v}(x, t)\right)^{T},
$$

characterizing the small deviations from the reference state $\mathbf{U}$, with the small perturbation $\xi_{i}^{\varphi}(x, t)$ of the potential $\varphi(x, t)$ being explicitly defined by the distribution of the density of the electron beam $\rho(x, t)$ and its small perturbation $\xi_{i}^{\rho}(x, t)$.

The behavior of Pierce diode with $i$-th perturbation of the state $\mathbf{U}$ is described by the following system

$$
\hat{L}\left(\mathbf{U}+\mathbf{V}_{i}\right)=0
$$

with the boundary conditions

$$
\begin{aligned}
& \rho(0, t)+\xi_{i}^{\rho}(0, t)=1, \\
& v(0, t)+\xi_{i}^{v}(0, t)=1, \\
& \varphi(0, t)+\xi_{i}^{\varphi}(0, t)=0, \\
& \varphi(1, t)+\xi_{i}^{\varphi}(1, t)=0 .
\end{aligned}
$$


Having linearized the system (19) we can rewrite the equations (19), (20) in the following way

$$
\partial \hat{L}\left(\mathbf{U}, \mathbf{V}_{i}\right)=0
$$

where $\partial \hat{L}\left(\mathbf{U}, \mathbf{V}_{i}\right)$ is the evolution operator $\hat{L}(\cdot)$ linearized near the reference state $\mathbf{U}(x, t)$. It describes the character of the development of the perturbations $\mathbf{V}_{i}$ with time. For the system (12)-(14) the evolution operator $\partial \hat{L}\left(\mathbf{U}, \mathbf{V}_{i}\right)$ can be written in the following way

$$
\begin{aligned}
& \frac{\partial^{2} \xi_{i}^{\varphi}}{\partial x^{2}}=-\alpha^{2} \xi_{i}^{\rho}, \\
& \frac{\partial \xi_{i}^{\rho}}{\partial t}=-\xi_{i}^{\rho} \frac{\partial v}{\partial x}-v \frac{\partial \xi_{i}^{\rho}}{\partial x}-\xi_{i}^{v} \frac{\partial \rho}{\partial x}-\rho \frac{\partial \xi_{i}^{v}}{\partial x}, \\
& \frac{\partial \xi_{i}^{v}}{\partial t}=-v \frac{\partial \xi_{i}^{v}}{\partial x}-\xi_{i}^{v} \frac{\partial v}{\partial x}-\frac{\partial \xi_{i}^{\varphi}}{\partial x}
\end{aligned}
$$

In this case the boundary conditions (20) for the small deviations $\mathbf{V}_{i}$ of the synchronous state are given by

$$
\begin{aligned}
& \xi_{i}^{\rho}(0, t)=0, \\
& \xi_{i}^{v}(0, t)=0, \\
& \xi_{i}^{\varphi}(0, t)=0, \\
& \xi_{i}^{\varphi}(1, t)=0 .
\end{aligned}
$$

Obtained operator $\partial \hat{L}(\mathbf{U}, \mathbf{V})$ is linear to relatively small deviations $\mathbf{V}$.

Now, taking into account the reference state $\mathbf{U}$, the perturbations $\mathbf{V}_{i}$, the evolution operator $\hat{L}(\mathbf{U})$ defined for system (12)-(14) with boundary conditions (15), and the linearization operator $\partial \hat{L}(\mathbf{U}, \mathbf{V})$ given by relations (22)-(23), we can calculate the spectrum of spatial Lyapunov exponents for the autonomous model of Pierce diode.

The behavior of autonomous model of Pierce diode is known to be defined by the only one dimensionless control parameter $\alpha$ called Pierce parameter. As we have mentioned above, Pierce diode is capable to demonstrate the different types of the complex spatio-temporal oscillations, with the transition from the periodic to chaotic ones being performed via the period doubling bifurcation cascade [18, 19, 22, 24, 25] when the $\alpha$-parameter value decreases in the range $\alpha \in(2.862 \pi, 2.88 \pi)$. Further decrease of the parameter $\alpha$ results in the considerable complication of the chaotic regime being realized in system (12)-(14) attended by the disappearance of the well-defined time scale of the spatio-temporal oscillations of the electron beam with the further transition to the wide-band chaotic spatio-temporal oscillations.

To characterize the type of dynamical regime being realized in the system under study we compute the spectrum of spatial Lyapunov exponents. In Fig. $2 a$ the dependence of the four highest spatial Lyapunov exponents on the parameter $\alpha$ is shown. To calculate the values of the spectrum of the spatial Lyapunov exponents $M=2 \times 10^{4}$ iterations of the renormalization and orthogonalizaiton have been done for each value of the control
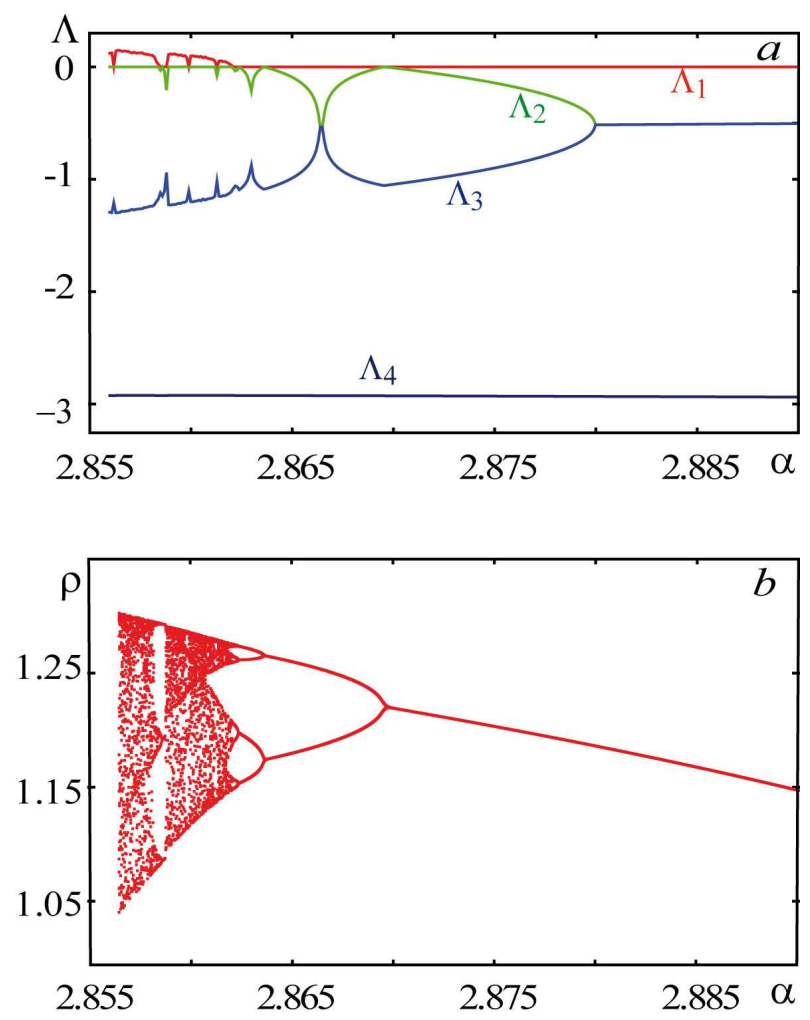

FIG. 2: (Color online) (a) The dependence of the four highest Lyapunov exponents and (b) bifurcation diagram of oscillations in the autonomous model of Pierce diode with $\alpha$ parameter value increasing

parameter $\alpha$, with the time interval between iterations being equal to $T=0.6$. In parallel, the bifurcation diagram of the density of the electron beam oscillations at the point of the interaction space $x=0.2$ is also indicated in Fig. 26 . It is easily seen from Fig. 2 that the spectrum of the highest spatial Lyapunov exponents allows to detect clearly the type of the dynamical regime realized in the system under study. For all values of the control parameter $\alpha$ one zero Lyapunov exponent corresponding to the system perturbations along the time axis is presented in the spectrum. In the region of the periodic oscillations (e.g., $\alpha \lesssim 2.862$ ) such zero Lyapunov exponent is the highest one, i.e., the chaotic dynamics is absent. All other spatial Lyapunov exponents are negative. At the same time, it is clearly seen that in the bifurcation points corresponding to the period doubling cascade (see, e.g., $\alpha \simeq 2.87$ ) the second Lyapunov exponent $\Lambda_{2}$ is also equal to zero, that indicates the qualitative changes in the system behavior with the control parameter varying as well as for the low-dimensional dynamical systems.

With the further decrease of the parameter $\alpha$ (see Fig. 2) the highest spatial Lyapunov exponent $\Lambda_{1}$ becomes positive, i.e. the transition to chaotic oscillations takes place. The second Lyapunov exponent remains to be the zero one. All others Lyapunov exponents are negative. Note, that in the periodicity windows, where the 
periodic oscillations take place, the positive Lyapunov exponent becomes also negative and the zero Lyapunov exponent gets to be the highest one.

It is important to note that in the case of the chaotic oscillations the system under study has the only one positive Lyapunov exponent. At the same time, the spatially extended media may theoretically have several positive Lyapunov exponents. The presence of the only one positive Lyapunov exponent is the evidence of the simplicity of chaotic oscillations being realized in the spatially extended system. One can assume that just due to that fact it has been possible to construct the finitedimensional model of the dynamics of the electron beam in the Pierce diode being the system of ordinary differential equations [20] by means of Galerkin method 23].

So, the spatial Lyapunov exponents could be easily applied for the detection and classification of the dynamical regimes being realized in the beam-plasma systems with the overcritical current. However, the field of the possible applications of the spectrum of the spatial Lyapunov exponents is not restricted to the consideration of the autonomous system. In particular, Lyapunov exponents are an effective tool for the analysis of the different types of chaotic synchronization being realized both in the finiteand infinite-dimensional systems [26 28]. Therefore, we consider the spectrum of spatial Lyapunov exponents for two unidirectionally coupled Pierce diodes in the next subsection.

\section{B. Two coupled Pierce diodes}

The system under study in this case is given by the partial differential equations

$$
\begin{gathered}
\frac{\partial^{2} \varphi^{1,2}}{\partial x^{2}}=-\left(\alpha^{1,2}\right)^{2}\left(\rho^{1,2}-1\right) \\
\frac{\partial \rho^{1,2}}{\partial t}=-\frac{\partial\left(\rho^{1,2} v^{1,2}\right)}{\partial x} \\
\frac{\partial v^{1,2}}{\partial t}=-v^{1,2} \frac{\partial v^{1,2}}{\partial x}+\frac{\partial \varphi^{1,2}}{\partial x}
\end{gathered}
$$

with the boundary conditions

$$
v^{1,2}(0, t)=1, \quad \rho^{1,2}(0, t)=1, \quad \varphi^{1,2}(0, t)=0,
$$

where the top indexes " 1 " and " 2 " correspond to the first and second coupled beam-plasma systems, respectively.

The unidirectional coupling between considered Pierce diodes is realized by the modification of the boundary conditions on the right boundary of the second (response) system, exactly in the same way as it has been done in [12]. In other words, the boundary conditions for the system (24) - 26) describing the dynamics of unidirectionally coupled Pierce diodes should be written as

$$
\left\{\begin{array}{l}
\varphi^{1}(1, t)=0 \\
\varphi^{2}(1, t)=\varepsilon\left(\rho^{2}(x=1, t)-\rho^{1}(x=1, t)\right),
\end{array}\right.
$$

i.e., the first (drive " 1 ") system is in the regime of autonomous oscillations influencing on the second (response "2") one.

To analyze the behavior of two coupled Pierce diodes we compute spatial Lyapunov exponents for the system (24) - (26). As a system state we use now the vector

$$
\mathbf{U}=\left(\rho^{1}(x, t), v^{1}(x, t), \rho^{2}(x, t), v^{2}(x, t)\right)^{T} .
$$

According to Eq. (5) the scalar product of the system states in this case is defined as

$$
\begin{aligned}
\left(\mathbf{U}_{\mathbf{1}}, \mathbf{U}_{\mathbf{2}}\right)= & \int_{0}^{1}\left(\rho_{1}^{1}(x, t) \rho_{2}^{1}(x, t)+v_{1}^{1}(x, t) v_{2}^{1}(x, t)\right) d x+ \\
& \int_{0}^{1}\left(\rho_{1}^{2}(x, t) \rho_{2}^{2}(x, t)+v_{1}^{2}(x, t) v_{2}^{2}(x, t)\right) d x .
\end{aligned}
$$

In Eq. (30) the integration is performed on the whole length of the considered system as well as in the case of Eq. (17). Equations (24)-(26) with the boundary conditions (27)- 28) for two unidirectionally coupled Pierce diodes play the role of the evolution operator (10).

By analogy with the relation (18) we use vectors

$$
\mathbf{V}_{i}=\left(\xi_{i}^{1 \rho}(x, t), \xi_{i}^{1 v}(x, t), \xi_{i}^{2 \rho}(x, t), \xi_{i}^{2 v}(x, t)\right)^{T},
$$

as the perturbations for two unidirectionally coupled Pierce diodes. They characterize the small deviations from the reference state $\mathbf{U}$, with the small perturbations $\xi_{i}^{1 \varphi}(x, t)$ and $\xi_{i}^{2 \varphi}(x, t)$ of the potentials $\varphi^{1,2}(x, t)$ as well as in the case of autonomous Pierce diode being explicitly defined by the distributions of the density $\rho^{1,2}(x, t)$ of the electron beam and its small perturbations $\xi_{i}^{1,2 \rho}(x, t)$.

For the system under study (24)-(28) the operator $\partial \hat{L}\left(\mathbf{U}, \mathbf{V}_{i}\right)$ defining the evolution of the small perturbations $\mathbf{V}_{i}$ of the reference state $\mathbf{U}$ is written in the form

$$
\begin{aligned}
\frac{\partial^{2} \xi_{i}^{1,2 \varphi}}{\partial x^{2}}= & -\left(\alpha^{1,2}\right)^{2} \xi_{i}^{1,2 \rho}, \\
\frac{\partial \xi_{i}^{1,2 \rho}}{\partial t}= & -\xi_{i}^{1,2 \rho} \frac{\partial v^{1,2}}{\partial x}-v^{1,2} \frac{\partial \xi_{i}^{1,2 \rho}}{\partial x}- \\
& -\xi_{i}^{1,2 v} \frac{\partial \rho^{1,2}}{\partial x}-\rho^{1,2} \frac{\partial \xi_{i}^{1,2 v}}{\partial x}, \\
\frac{\partial \xi_{i}^{1,2 v}}{\partial t}= & -v^{1,2} \frac{\partial \xi_{i}^{1,2 v}}{\partial x}-\xi_{i}^{1,2 v} \frac{\partial v^{1,2}}{\partial x}-\frac{\partial \xi_{i}^{1,2 \varphi}}{\partial x}
\end{aligned}
$$

with the boundary conditions for the small deviations $\mathbf{V}_{i}$ from the reference state being the following

$$
\begin{aligned}
& \xi_{i}^{1,2 \rho}(0, t)=0 \\
& \xi_{i}^{1,2 v}(0, t)_{i}=0 \\
& \xi_{i}^{1,2 \varphi}(0, t)=0 \\
& \xi_{i}^{1 \varphi}(1, t)=0 \\
& \xi_{i}^{2 \varphi}(1, t)=\varepsilon\left(\xi_{i}^{2 \rho}(1, t)-\xi_{i}^{1 \rho}(1, t)\right) .
\end{aligned}
$$

So, the computations of the spectrum of the spatial Lyapunov exponents for two unidirectionally coupled 


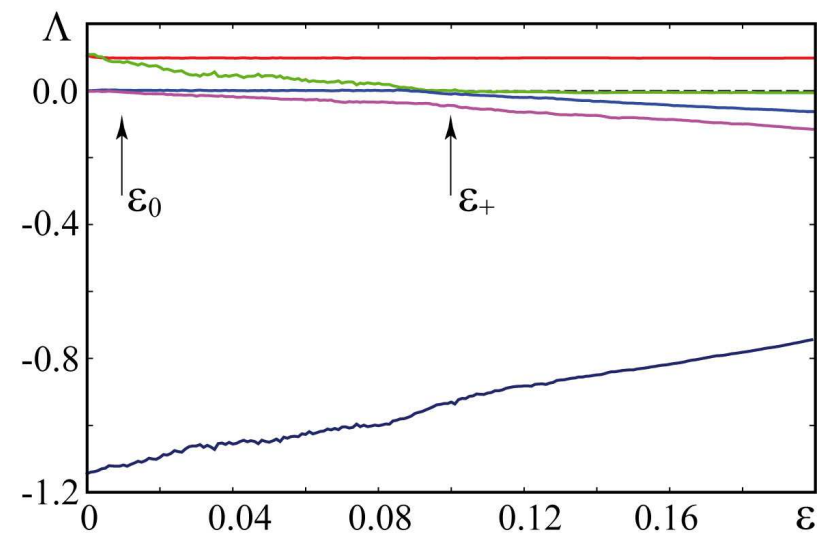

FIG. 3: (Color online) The dependence of the five highest Lyapunov exponents on the coupling parameter $\varepsilon$ for two unidirectionally coupled Pierce diodes

Pierce diodes is performed in the same way as for the autonomous system with the only one difference consisting in the fact that the system state $\mathbf{U}$, small perturbations $\mathbf{V}_{i}$, the scalar product $\left(\mathbf{U}_{1}, \mathbf{U}_{2}\right)$ and the evolution operators $\hat{L}(\mathbf{U})$ and $\partial \hat{L}\left(\mathbf{U}, \mathbf{V}_{i}\right)$ are defined for two interacting systems.

The dependence of the five highest spatial Lyapunov exponents on the coupling parameter strength $\varepsilon$ for two unidirectionally coupled Pierce diodes (24)-(26) with the boundary conditions (27)-(28) is shown in Fig. 3. The control parameter values $\alpha^{1}=2.858, \alpha^{2}=2.860$ have been chosen in such a way that the autonomous dynamics of each Pierce diode is being chaotic one. It is clearly seen that in the absence of the coupling $\varepsilon=0$ two highest Lyapunov exponents are positive (that is the evidence of the presence of the chaotic dynamics in each Pierce diode), two Lyapunov exponents are equal to zero (that corresponds to the perturbations of the system states along the time axis), the fifth and others Lyapunov exponents are negative.

With the coupling parameter value increase the quantitative values of the Lyapunov exponents start changing. Due to the unidirectional type of the coupling between Pierce diodes the spectrum of Lyapunov exponents $\Lambda_{1} \geq \Lambda_{2} \geq \ldots$ can be divided into two parts. One of them corresponds to the Lyapunov exponents calculated for the drive system $\Lambda_{1}^{1} \geq \Lambda_{2}^{1} \geq \ldots$ and due to the independency of the behavior of the drive system on the response one they do not depend on the coupling parameter strength. The second part is the conditional Lyapunov exponents, i.e., Lyapunov exponents computed for the response system. They depend on the coupling parameter strength $\varepsilon$ and, therefore, are called conditional.

Such a model of Pierce diode is known to demonstrate the different regimes of chaotic synchronization, including complete, generalized and time scale synchronization [12]. The occurrence of any type of the synchronous regime should be accompanied by the transformations in the spectrum of Lyapunov exponents. In particular, for the systems with a small number of degrees of freedom the transition of the zero conditional (corresponding to the response system) Lyapunov exponent in the field of the negative values precedes the phase or time scale synchronization regimes in the case of the small values of the parameter detuning [28 30]. When the highest conditional Lyapunov exponent passes through zero, the generalized synchronization regime takes place [12, 31].

The very same phenomena is observed for two coupled spatially extended beam plasma systems. When the coupling parameter value increases the response system becomes synchronized by the drive one, with the qualitative changes in the spectrum of the conditional Lyapunov exponents taking place. In Fig. 3 two critical points $\varepsilon_{0} \approx 0.01$ and $\varepsilon_{+} \approx 0.09$ are clearly seen. As in the case of the low-dimensional system, the first of them corresponds to the transition of the zero conditional Lyapunov exponent in the field of the negative values, whereas the second one relates to the zero-pass of the positive conditional Lyapunov exponent. The negativeness of the largest conditional spatial Lyapunov exponent is the evidence of the generalized synchronization regime that has been confirmed with the help of the auxiliary system method [12, 32].

\section{SPECTRUM OF SPATIAL LYAPUNOV EXPONENTS FOR THE TRANSVERSE FIELD BACKWARD WAVE OSCILLATOR}

To illustrate the generality and efficiency of the proposed method, as well as their applicability for the widespread class of spatially-extended systems, we consider the behavior of the transverse field backward wave oscillator being the classical models of the electrons-wave systems, demonstrating the complex oscillations including the chaotic regimes as well as hyperchaos.

The dynamics of the backward wave oscillator is described by the system of the dimensionless equations

$$
\begin{gathered}
\frac{\partial F}{\partial t}-\frac{\partial F}{\partial x}=-A I \\
\frac{\partial I}{\partial x}+j|I|^{2} I=-A F
\end{gathered}
$$

where $F(x, t)$ is the dimensionless complex-valued amplitude of the electric field and $I(x, t)$ is the complexvalued current $(0<x<1)$. The control parameter $A$ can be considered as the dimensionless length of the system. The equations (34)-(35) have been integrated with the boundary conditions:

$$
F(1, t)=0, \quad I(0, t)=0 .
$$

To calculate the spectrum of Lyapunov exponents we define the main state of this system as

$$
\mathbf{U}=F(x, t) .
$$


It is important to note, that the distribution of the complex-valued current is excluded from the main system state, since it is explicitly defined by the electrical field $F(x, t)$.

The scalar product of complex-valued functions in this case is defined as follows

$$
\left(\mathbf{U}_{\mathbf{1}}, \mathbf{U}_{\mathbf{2}}\right)=\int_{0}^{1} F_{1}(x, t) F_{2}^{*}(x, t) d x,
$$

where the integration, as for the case of Pierce diode, should be performed over the whole length of the considered system.

The main system state (37) evaluates according to the operator (11), presented in the form of differential equations (34)-(35). These equations (34)-(35) have been integrated using the Lax-Wendroff method and the thirdorder Runge-Kutta method. The time and space integration steps have been selected as $\Delta x=0.004$ and $\Delta t=0.002$, respectively.

The state (37) plays the role of the reference state. To calculate $N$ largest spatial Lyapunov exponents we consider the set of perturbations

$$
\mathbf{V}_{i}=\left(\xi_{i}^{F}(x, t)\right)
$$

Again, the current has been eliminated from the consideration, since the small perturbations $\xi_{i}^{I}(x, t)$ of the complex-valued current $I(x, t)$ are explicitly defined by the distribution of the high-frequency electric field $F(x, t)$ and its small perturbations $\xi_{i}^{F}(x, t)$.

The evolution of the set of small perturbations is described by the linearized operator (8), which for the considered system can be written in the form:

$$
\begin{aligned}
& \frac{\partial \xi_{i}^{F}}{\partial t}-\frac{\partial \xi_{i}^{F}}{\partial x}=-A \xi_{i}^{I} \\
& \frac{\partial \xi_{i}^{I}}{\partial t}+2 j|I|^{2} \xi_{i}^{I^{*}}+j I^{2} \xi_{i}^{I}=-A \xi_{i}^{F}
\end{aligned}
$$

with the boundary conditions

$$
F(1, t)+\xi_{i}^{F}(1, t)=0, \quad I(0, t)+\xi_{i}^{I}(0, t)=0 .
$$

The Lyapunov sums and values of SLEs have been estimated with the help of expressions (10)-(11).

In Fig. 4, $a$ the dependence of the four highest spatial Lyapunov exponents on the parameter $A$ is shown. To give the information of the behavior of the lower SLEs the dependence of the 10th LE is also given in Fig. 4. The bifurcation diagram of the complex-valued amplitude of the electric field is presented in Fig. 4, $b$. The largest Lyapunov exponent $\lambda_{1}$ is equal to zero when the control parameter has the value being less then 1.83, that corresponds to the stationary state, when the antiphase oscillations of the real and imaginary parts of the complexvalued amplitude of electric field takes place, while its module remains constant. All other SLEs $\lambda_{2}, \lambda_{3}, \ldots$ are negative.

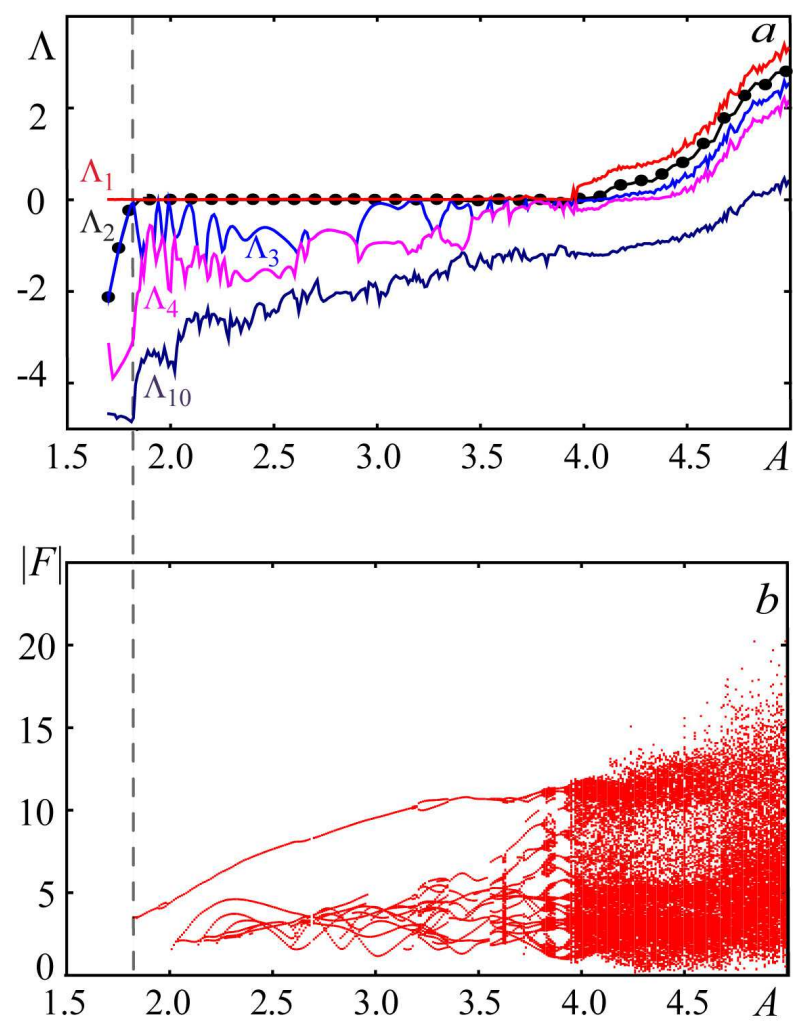

FIG. 4: (Color online) (a) The dependence of the four highest and the 10th Lyapunov exponents (the second LE is shown by points $(\bullet))$ and $(b)$ the bifurcation diagram of oscillations in the transverse field Backward wave oscillator with the $A$ parameter value increase

For the bifurcation point $A_{c} \approx 1.83$ the stationary state becomes unstable and, as a consequence, the periodic oscillations of the module of the complex-valued electric field $|F|$ are observed in the system under study. In the region of the periodic oscillations $A>A_{c}$ the second Lyapunov exponent $\lambda_{2}$ is also equal to zero. At the same time, all other Lyapunov exponents are negative, as before. Note also, at the bifurcation point $A_{c}$ the third Lyapunov exponent $\lambda_{3}$ is equal to zero, that indicates the qualitative changes taking place in the spatially-extended system.

With the further increase of the $A$-parameter the transition from periodic oscillations to chaotic dynamics is observed. This transformation is accompanied by the modification of the spectrum of SLEs, when the largest Lyapunov exponent becomes positive $\left(A_{c h} \approx 3.9\right)$. As for the case of Pierce diode, it can be used as a criterium of the transition to the chaotic regime, and, as well as for Pierce diode, this transformation of the SLEs spectrum agrees with the bifurcation diagram (Fig. 4, $a$ ).

As it has been described above, the chaotic regimes in the spatially-extendent systems, in general, can be characterized by several positive Lyapunov exponents. It is clearly seen from Fig. 4 , the number of the positive Lya- 
punov exponents increases with the grow of the value of $A$-parameter, with the chaotic regime (see bifurcation diagram, Fig. 4, a) becoming more complicated. Note also, as soon as one of the Lyapunov exponents being zero becomes positive, the largest negative Lyapunov exponent takes the value zero.

\section{THE SELECTION OF THE SYSTEM STATE: BRIEF DISCUSSION}

The proposed approach for the computation of the spectrum of the spatial Lyapunov exponents is shown to be the effective and powerful tool to study and analyze the complex behavior of the beam plasma systems as well as the other spatially extended media. Therefore, it is not surprised that this tool attracts great attention of scientists [33, 34]. Nevertheless, one have to be very careful computing the spectrum of the spatial Lyapunov exponents, since there may be certain particularities connected with the SLEs calculation which may result to the inaccurate or even incorrect decisions made and, therefore, they must be taken into account by scientists. In this section one of the possible problems will be discussed for the scientists to avoid the potential errors.

When the spectrum of spatial Lyapunov exponents is calculated the state $\mathbf{U}$ of the system should be chosen properly. In the studies given above certain variables (such as the potential $\varphi(x, t)$ in the case of Pierce diode or the current $I(x, t)$ in the case of the transverse field backward wave oscillator) have been excluded from the system state having based on the reason that these variables may be explicitly defined through the other ones which are included in the system state. To prove the validity of this approach we have also calculated the spectrum of the spatial Lyapunov exponents when the whole set of the variables is supposed to be the system state. For the Pierce diode the system state in this case is

$$
\mathbf{U}=(\varphi(x, t), \rho(x, t), v(x, t))^{T},
$$

whereas for the transverse field backward wave oscillator it is defined as

$$
\mathbf{U}=(F(x, t), I(x, t))^{T} .
$$

Fig. 5 illustrates the three highest Lyapunov exponents of Pierce diode obtained in both cases (when $\mathbf{U}=$ $(\varphi(x, t), \rho(x, t), v(x, t))^{T}$ and $\left.\mathbf{U}=(\rho(x, t), v(x, t))^{T}\right)$. One can see that the LEs correspond exactly to the values, calculated in Sec. II] Analogously, in Fig. 6] the first, the third and the fifth Lyapunov exponents corresponding to the periodical oscillations in the backward wave oscillator are shown. It is clearly seen, that the values of Lyapunov exponents obtained in the both cases $\left(\mathbf{U}=(F(x, t), I(x, t))^{T}\right.$ and $\left.\mathbf{U}=F(x, t)\right)$ are identical for all values of the dimensionless system length $A$.

So, certain variables can be excluded from the system state if they may be explicitly defined through the

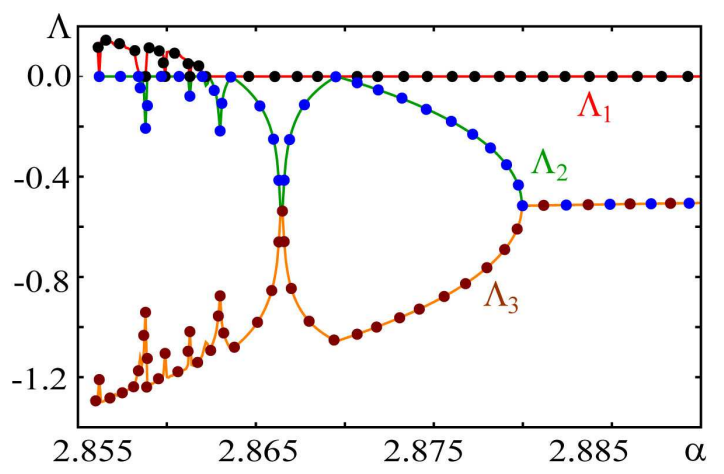

FIG. 5: (Color online) The three highest Lyapunov exponents of Pierce diode calculated both for $\mathbf{U}=(\varphi(x, t), \rho(x, t), v(x, t))^{T} \quad$ (points) and for $\mathbf{U}=(\rho(x, t), v(x, t))^{T}$ (lines)

other system variables in the every moment of time or, in other words, if the time derivatives of these variables are not presented in the evolution operator. Nevertheless, in general, the choice of the system state $\mathbf{U}$ requires the thoroughness and accuracy. Thus, the majority of the beam plasma and microwave systems are simulated with the help of the particle methods [35]. In this case the state $\mathbf{U}(x, t)$ of the system under study is determined by the electromagnetic field as well as locations and velocities of all particles used for simulation. As far as the electromagnetic field is concerned there is no problem to apply the renormalization and orthogonalizaiton procedures in the very same way as it has been described in Sec. [1, since this field is the function of the coordinate, i.e., $E(x, t) \in \mathbb{L}^{2}, B(x, t) \in \mathbb{L}^{2}$. On the contrary, the set of particles emulating the electron beam can not be renormalized and orthogonalized with the help of the technique given above, since each particle is characterized by its own coordinate and velocity. As a consequence, the scientists dealing with such a type of systems use only the electromagnetic field as the system state $\mathbf{U}(x, t)$ to avoid the uncertainty connected with particle dynamics evaluation (see, e.g. [33]). Although in some instances the obtained in such a way results seem to be plausible, we show below that this approach gives the very crude and inaccurate solution.

To illustrate this point we consider the emulation of the spatial Lyapunov exponent calculation for the spatially extended system simulated by the particle method by the example of Pierce diode. Since in this case the electron beam ("particles") can not be used when the renormalization and orthogonalizaiton procedures are implemented, we consider the truncated system state

$$
\mathbf{U}^{\prime}(x, t)=v(x, t)
$$

in parallel with (16). The dynamics of Pierce diode state and its perturbations are evaluated according to the operator (12)-(14), whereas the Gramm-Schmidt procedure is realized only for the truncated state (44) that excludes the beam of electrons from the renormalization and or- 


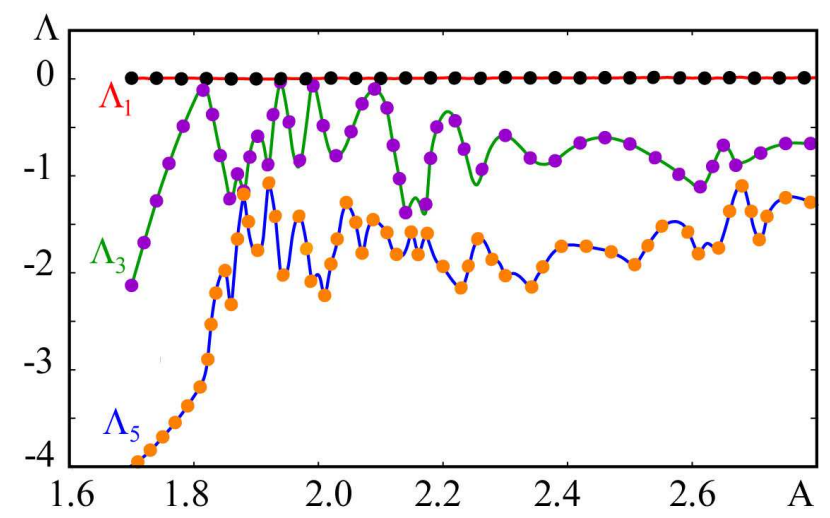

FIG. 6: (Color online) The first, the third and the fifth highest Lyapunov exponents of the backward wave oscillator calculated both for $\mathbf{U}=(F(x, t), I(x, t))^{T}$ (points) and $\mathbf{U}=F(x, t)$ (lines)

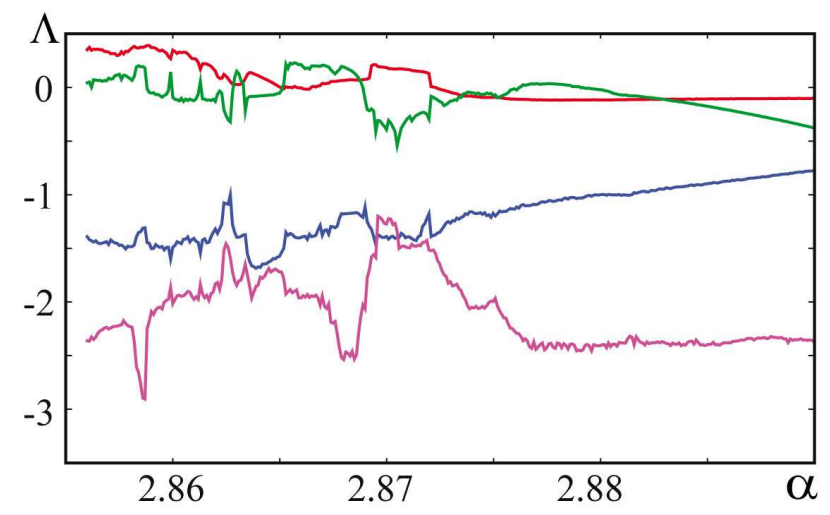

FIG. 7: (Color online) The four highest Lyapunov exponents calculated for the case when the beam of electrons is excluded from the renormalization and orthogonalizaiton procedure (compare with Fig. 2 a)

thogonalizaiton, exactly in the same way, as it has been done, e.g., in [33] for the system simulated by the particle method.

The four largest Lyapunov exponents (solid lines) calculated for Pierce diode by means of the described technique (it models the approach used for the system simulated by particle method) are shown in Fig. [7 to be compared with the Lyapunov exponents calculated in Sec. IIA (see Fig. 2). It is easy to see that the true dependence of the spatial Lyapunov exponents (Fig. 2) is seriously distorted (Fig. 7) when the electron beam is excluded from the renormalization and orthogonalizaiton procedures. E.g., even zero Lyapunov exponent being known to have always to exist in the autonomous system (since it corresponds to the perturbation along with the time axis) is not calculated properly in comparison with the case, when all sufficient quantities (in the considered case these are $\rho(x, t)$ and $v(x, t))$ are taken into account.

Evidently, for some values of the control parameter $\alpha$ the obtained set of spatial Lyapunov exponents seems to be reasonable. E.g., for $\alpha=2.856$ the largest value of the calculated spatial Lyapunov exponent spectrum is positive that corresponds to the chaotic dynamics observed in the system, the second Lyapunov exponent is close to zero that also agrees well with the theory, the other Lyapunov exponents are negative. So, if the careful verification of the obtained results has not been done these results may be assumed to be correct, although the values of the calculated spatial Lyapunov exponents differ greatly from the true ones. Moreover, for the other values of the control parameters the calculated data may be obviously contradictory (see, e.g., $\alpha=2.87$, where despite of the periodic regime the positive value of the spatial Lyapunov exponent is obtained, whereas the zero Lyapunov exponent is absent). The inconsistency of the obtained values of the spatial Lyapunov exponents with the system dynamics may be revealed with the help of the examination of the dependence of SLEs on the system control parameter value, but, typically, this analysis is not given.

Therefore, we can come to the conclusion that the calculation of the spatial Lyapunov exponents for the models simulated by the particle methods must be used very carefully. Unfortunately, at present there is no methods allowing the calculations of SLEs for such kind of the dynamical systems and this problem should be solved in the future.

\section{Conclusion}

In the present work we have proposed the method for the calculation of the spectrum of the spatial Lyapunov exponents for the spatially extended beam plasma systems. This method is shown to be effective and powerful tool for the analysis of the complex behavior of the beam plasma systems as well as the other spatially extended systems. The possible applications of the spectrum of the spatial Lyapunov exponents are not restricted to the consideration of the autonomous system, e.g., SLEs are the effective tool for the analysis of the different types of chaotic synchronization. At the same time, the calculation of the spatial Lyapunov exponents for the models simulated by the particle methods must be used very carefully, whereas the problem of calculations of SLEs for these systems should be solved in the future.

Though the calculation of the spectrum of the spatial Lyapunov exponents has been illustrated with the help of several examples, we expect that this technique may be used in many other relevant circumstances, as e.g. laser systems [36], semiconductor superlattices [37], etc. 
[1] Y. Bogomolov and A. D. Yunakovskii, J. Comput. Phys. 58, 80 (1985).

[2] C. Chen and R. C. Davidson, Phys. Rev. A 43, 5541 (1991).

[3] V. A. Goryashko, K. Ilyenko, and A. H. Opanasenko, Physical Review Special Topics Accelerators and Beams 12, 100701 (2009).

[4] T. Klinger, C. Schroder, D. Block, F. Greiner, A. Piel, G. Bonhomme, and V. Naulin, Phys.Plasmas 8, 1961 (2001).

[5] A. E. Hramov, A. A. Koronovskii, and I. S. Rempen, Chaos 16, 013123 (2006).

[6] T. S. Goud, R. Ganesh, Y. C. Saxena, D. Raju, K. Sathyanarayana, K. K. Mohandas, and C. Chavda, Physics of Plasmas 19, 032307 (pages 12) (2012).

[7] D. L. Toufen, G. Z. O. a, I. L. Caldas, F. A. Marcus, and K. W. Gentle, Physics of Plasmas 19, 012307 (pages 8) (2012).

[8] T. Klinger, A. Latten, A. Piel, E. Bonhomme, and T. Pierre, Plasma Phys. Control. Fusion 39, 145 (1997).

[9] C. M. Ticos, E. Rosa, W. B. Pardo, J. A. Walkenstein, and M. Monti, Phys. Rev. Lett. 85, 2929 (2000).

[10] D. Block, A. Piel, C. Schröder, and T. Klinger, Phys. Rev. E 63, 056401 (2001).

[11] A. E. Hramov, A. A. Koronovskii, P. V. Popov, and I. S. Rempen, Chaos 15, 013705 (2005).

[12] R. A. Filatov, A. E. Hramov, and A. A. Koronovskii, Phys. Lett. A 358, 301 (2006).

[13] K. Thamilmaran, D. V. Senthilkumar, A. Venkatesan, and M. Lakshmanan, Phys. Rev. E 74, 036205 (2006).

[14] R. Porcher and G. Thomas, Phys. Rev. E 64, 010902(R) (2001).

[15] T. E. Karakasidis, A. Fragkou, and A. Liakopoulos, Phys. Rev. E 76, 021120 (2007).

[16] R. M. Dünki, Phys. Rev. E 62, 6505 (2000).

[17] J. R. Pierce, J.Appl.Phys. 15, 721 (1944).

[18] B. B. Godfrey, Phys. Fluids 30, 1553 (1987).
[19] H. Matsumoto, H. Yokoyama, and D. Summers, Phys.Plasmas 3, 177 (1996).

[20] D. I. Trubetskov and A. E. Hramov, Lectures on microwave electronics for physicists (In Russian) (Vol. 1. Fizmatlit, Moscow, 2003).

[21] S. Kuhn, Contrib. Plasma Phys 34, 495 (1994).

[22] S. Kuhn and A. Ender, J.Appl.Phys. 68, 732 (1990).

[23] P. J. Rouch, Computational fluid dynamics (Hermosa publishers, Albuquerque, 1976).

[24] P. A. Lindsay, X. Chen, and M. Xu, Int. J.Electronics 79, 237 (1995).

[25] A. E. Hramov and I. S. Rempen, Int. J.Electronics 91, 1 (2004).

[26] K. Pyragas, Phys. Rev. E 56, 5183 (1997).

[27] A. E. Hramov, A. A. Koronovskii, and O. I. Moskalenko, Europhysics Letters 72, 901 (2005).

[28] A. E. Hramov, A. A. Koronovskii, and M. K. Kurovskaya, Phys. Rev. E 78, 036212 (2008).

[29] M. G. Rosenblum, A. S. Pikovsky, and J. Kurths, Phys. Rev. Lett. 78, 4193 (1997).

[30] A. E. Hramov, A. A. Koronovskii, and M. K. Kurovskaya, Phys. Rev. E 75, 036205 (2007).

[31] K. Pyragas, Phys. Rev. E 54, R4508 (1996).

[32] H. D. Abarbanel, N. F. Rulkov, and M. M. Sushchik, Phys. Rev. E 53, 4528 (1996).

[33] S. P. Kuznetsov and D. I. Trubetskov, Radiophysics and Quantum Electronics 47, 341 (2004).

[34] A. E. Hramov, A. A. Koronovskii, and P. V. Popov, Phys. Rev. E 77, 036215 (2008).

[35] R. W. Hockney and J. W. Eastwood, Computer simulation using particles (NY: McGraw-Hill, 1981).

[36] S. Boccaletti, E. Allaria, R. Meucci, and F. T. Arecchi, Phys. Rev. Lett. 89, 194101 (2002).

[37] M. T. Greenaway, A. G. Balanov, E. Schöll, and T. M. Fromhold, Phys. Rev. B 80, 205318 (2009). 